\title{
COMPLEX NUMBERS SIMILAR TO THE GENERALIZED BERNOULLI NUMBERS AND THEIR APPLICATIONS
} BRAHIM MITTOU $^{1^{*}}$ and ABDALLAH DERBAL ${ }^{2}$

${ }^{1}$ Univercity Kasdi Merbah Ouargla, EDPNL \& HM Laboratory of ENS Kouba, Algiers, Algeria ${ }^{2}$ ENS Old Kouba, EDPNL \& HM Laboratory of ENS Kouba, Algiers, Algeria

*Corresponding author.E-mail: mathmittou@gmail.com

\section{DOI: 10.20948/mathmontis-2021-50-2}

Summary. Let $\chi$ be a primitive Dirichlet character modulo $k \geq 3$. In this paper, we define complex numbers associated with $\chi$, which we denote by $C_{r}(\chi)(r=0,1, \ldots)$, and we discuss their properties and their relationships with the generalized Bernoulli numbers.

\section{INTRODUCTION}

Let $\chi$ be a Dirichlet character modulo $k \geq 3$. Then the classical generalized Bernoulli numbers $B_{m}(\chi)$ for $(m=0,1, \ldots)$ are defined by:

$$
\sum_{l=1}^{k} \chi(l) \frac{z e^{l z}}{e^{k z}-1}=\sum_{m=0}^{+\infty} B_{m}(\chi) \frac{z^{m}}{m !}, \quad|z|<\frac{2 \pi}{k}
$$

They can be expressed in terms of Bernoulli polynomials as (see [2, formula (4.1)]):

$$
B_{m}(\chi)=k^{m-1} \sum_{l=1}^{k} \chi(l) B_{m}\left(\frac{l}{k}\right),
$$

where the Bernoulli polynomials $B_{m}(x)$ are defined by:

$$
\frac{z e^{x z}}{e^{z}-1}=\sum_{m=0}^{+\infty} B_{m}(x) \frac{z^{m}}{m !}, \quad|z|<2 \pi .
$$

The Dirichlet L-function corresponding to $\chi$ is defined by:

$$
L(s, \chi)=\sum_{n=1}^{+\infty} \frac{\chi(n)}{n^{s}}, \quad \mathfrak{R}(s)>1 .
$$

Now, let $\chi$ be a primitive character. It is well known [2, Theorem 9.10] that the values of $L(s, \chi)$ at $s=-n,(n=0,1, \ldots)$ can be expressed by the generalized Bernoulli numbers as:

$$
L(-n, \chi)=-\frac{B_{n+1}(\chi)}{n+1} \text {. }
$$

Also from [2, Theorem 9.6] if $\chi(-1)=(-1)^{n}(n=1,2, \ldots)$, then the special values of $L(s, \chi)$ at $s=n$ are given by:

$$
L(n, \chi)=(-1)^{n-1} \frac{\tau(\chi)}{2 n !}\left(\frac{2 \pi i}{k}\right)^{n} B_{n}(\bar{\chi})
$$

where $\tau(\chi)=\sum_{a=1}^{k} \chi(a) e^{\frac{2 \pi i a}{k}}$ is the Gaussian sum associated with $\chi$. 
In this paper, based on a definition given by Davies and Haselgrove in [4], we rewrite the formulas (1.1) and (1.2) in terms of the numbers $C_{r}(\chi)$. We also give some results, properties and applications of these numbers, and their relationship with the generalized Bernoulli numbers.

\section{DEFINITION AND LEMMAS}

In order to prove our main results, we give the following definition and we need the later lemmas.

Definition 2.1 Let $\chi$ be a non-principal character modulo $k \geq 3$. For an integer $r \geq 0$ we define the function $p_{r}(x, \chi)$ for $x \in \mathbb{R}_{+}$as follows:

$$
\begin{gathered}
p_{0}(x, \chi)=S(x, \chi)+C_{0}(\chi), \text { where } S(x, \chi)=\sum_{n \leq x} \chi(n), \\
p_{r+1}(x, \chi)=\int_{0}^{x} p_{r}(t, \chi) d t+C_{r+1}(\chi), \\
\int_{0}^{k} p_{r}(x, \chi) d x=0 .
\end{gathered}
$$

Lemma 2.2 Let $\chi$ be a non-principal character modulo $k \geq 3$. Then

1. The function $S(x, \chi)$ is $k$-periodic.

2. For any function $f$ defined from $[0, k)$ to $\mathbb{C}$ which has an antiderivative $g$ we have

$$
\int_{0}^{k} S(t, \chi) f(t) d t=-\sum_{m=1}^{k-1} g(m) \chi(m) .
$$

3. If $\chi$ is primitive, then the Fourier series expansion of $S(x, \chi)$ is given by:

$$
\begin{aligned}
& S(x, \chi)=\frac{1}{k} \sum_{m=1}^{k-1} m \chi(m)+\frac{E(\chi)}{\pi} \sum_{n=1}^{+\infty} \frac{\bar{\chi}(n)}{n} \sin \left(\frac{2 n \pi x}{k}\right) \text { if } \chi(-1)=+1, \\
& S(x, \chi)=\frac{1}{k} \sum_{m=1}^{k-1} m \chi(m)-\frac{E(\chi)}{\pi} \sum_{n=1}^{+\infty} \frac{\bar{\chi}(n)}{n} \cos \left(\frac{2 n \pi x}{k}\right) \text { if } \chi(-1)=-1,
\end{aligned}
$$

where

$$
E(\chi)=\sum_{m=1}^{k} \chi(m){ }_{\sin }^{\cos }\left(\frac{2 m \pi}{k}\right) \text { if } \chi(-1)= \pm 1
$$

Proof. 1. For any integer $N$, we have

$$
S(x+N k, \chi)=\sum_{n \leq x+N k} \chi(n)=\sum_{n \leq x} \chi(n+N k)=\sum_{n \leq x} \chi(n)=S(x, \chi),
$$

so $S(x, \chi)$ is $k$-periodic. 
2. This follows at once from the integration by parts and the fact that $\sum_{m=1}^{k-1} \chi(m)=0$ (see e.g. $[1$, p. 30]).

3. The Fourier series expansion of $S(x, \chi)$ is given by:

where

$$
a_{0}+\sum_{n=1}^{+\infty}\left(a_{n} \cos \left(\frac{2 n \pi x}{k}\right)+b_{n} \sin \left(\frac{2 n \pi x}{k}\right)\right)
$$

$$
\begin{gathered}
a_{0}=\frac{1}{k} \int_{0}^{k} S(t, \chi) d t=\frac{-1}{k} \sum_{m=1}^{k-1} m \chi(m), \\
a_{n}=\frac{2}{k} \int_{0}^{k} S(t, \chi) \cos \left(\frac{2 n \pi t}{k}\right) d t=\frac{-1}{n \pi} \sum_{m=1}^{k-1} \chi(m) \sin \left(\frac{2 m \pi x}{k}\right) \text { for } n \geq 1, \\
b_{n}=\frac{2}{k} \int_{0}^{k} S(t, \chi) \sin \left(\frac{2 n \pi t}{k}\right) d t=\frac{1}{n \pi} \sum_{m=1}^{k-1} \chi(m) \cos \left(\frac{2 m \pi x}{k}\right) \text { for } n \geq 1 .
\end{gathered}
$$

If $\chi(-1)=+1$, we put $\theta=\frac{2 n \pi}{k}$ and we show that $a_{n}=0$ for $n \geq 1$. Indeed

$$
\begin{aligned}
a_{n}=\frac{-1}{n \pi} \sum_{m=1}^{k-1} \chi(m) \sin (m \theta) & =\frac{-1}{n \pi} \sum_{m=1}^{k-1} \chi(k-m) \sin ((k-m) \theta) \\
& =\frac{1}{n \pi} \sum_{m=1}^{k-1} \chi(m) \sin (m \theta)=-a_{n} .
\end{aligned}
$$

Also $a_{0}=0$, since

$$
\sum_{m=1}^{k-1} m \chi(m)=\sum_{m=1}^{k-1}(k-m) \chi(k-m)=k \sum_{m=1}^{k-1} \chi(m)-\sum_{m=1}^{k-1} m \chi(m)=-\sum_{m=1}^{k-1} m \chi(m) .
$$

If $\chi(-1)=-1$, we show by the same way that $b_{n}=0$ for $n \geq 1$.

According to [1, Theorem 8.15] we have

$$
\bar{\chi}(n) E(\chi)=\sum_{m=1}^{k} \chi(m) \frac{\cos }{\sin }\left(\frac{2 m n \pi}{k}\right) \text { if } \chi(-1)= \pm 1
$$

from which we can write for $n \geq 1$ :

$$
b_{n}=\frac{1}{n \pi} \times E(\chi) \bar{\chi}(n) \text { if } \chi(-1)=+1, \text { and } a_{n}=\frac{-1}{n \pi} \times E(\chi) \bar{\chi}(n) \text { if } \chi(-1)=-1 .
$$

This completes the proof.

Lemma 2.3 Let $\chi$ be a primitive character modulo $k \geq 3$. Then

1 . For $r \geq 1$, the function $p_{r}(x, \chi)$ is continuous and $k$-periodic.

2. The Fourier series expansion of $p_{r}(x, \chi)$ is given by: 


$$
p_{0}(x, \chi)= \pm \frac{E(\chi)}{\pi} \sum_{n=1}^{+\infty} \frac{\bar{\chi}(n)}{n} \sin \left(\frac{2 n \pi x}{k}\right) \text { if } \chi(-1)= \pm 1
$$

For $r \geq 1$ and $\chi(-1)=+1$, we have

$$
\begin{gathered}
p_{2 r-1}(x, \chi)=(-1)^{r} \frac{E(\chi)}{\pi}\left(\frac{k}{2 \pi}\right)^{2 r-1} \sum_{n=1}^{+\infty} \frac{\bar{\chi}(n)}{n^{2 r}} \cos \left(\frac{2 n \pi x}{k}\right), \\
p_{2 r}(x, \chi)=(-1)^{r} \frac{E(\chi)}{\pi}\left(\frac{k}{2 \pi}\right)^{2 r} \sum_{n=1}^{+\infty} \frac{\bar{\chi}(n)}{n^{2 r+1}} \sin \left(\frac{2 n \pi x}{k}\right) .
\end{gathered}
$$

For $r \geq 1$ and $\chi(-1)=-1$, we have

$$
\begin{aligned}
& p_{2 r-1}(x, \chi)=(-1)^{r} \frac{E(\chi)}{\pi}\left(\frac{k}{2 \pi}\right)^{2 r-1} \sum_{n=1}^{+\infty} \frac{\bar{\chi}(n)}{n^{2 r}} \sin \left(\frac{2 n \pi x}{k}\right), \\
& p_{2 r}(x, \chi)=(-1)^{r+1} \frac{E(\chi)}{\pi}\left(\frac{k}{2 \pi}\right)^{2 r} \sum_{n=1}^{+\infty} \frac{\bar{\chi}(n)}{n^{2 r+1}} \cos \left(\frac{2 n \pi x}{k}\right) .
\end{aligned}
$$

Proof. 1. It is clear that, for $r \geq 1$ the function $p_{r}(x, \chi)$ is continuous, since it is the primitive of piece-wise continuous function. The properties of the Dirichlet characters and formulas (2.1) allow us to show by induction that, for any $N \in \mathbb{Z}$ and $r \in \mathbb{N}$ :

$$
\int_{N k}^{(N+1) k} p_{r}(x, \chi) d x=0, \text { and } p_{r}(x+N k, \chi)=p_{r}(x, \chi),
$$

from which the periodicity of $p_{r}(x, \chi)$ follows.

2. The formulas (2.3), (2.4) and (2.5) are obtained by successive integrations of (2.2), taking into consideration the formulas (2.1). The lemma is proved.

\section{MAIN RESULTS}

In this section we give our main results. Let us start by the explicit formulas for the numbers $C_{r}(\chi)$.

Theorem 3.1 Let $\chi$ be a primitive character modulo $k \geq 3$. Then the numbers $C_{r}(\chi)$ are explicit as follows:

If $\chi(-1)=+1$,

$$
C_{1}(\chi)=\frac{-1}{2 k} \sum_{m=1}^{k-1} m^{2} \chi(m), \quad C_{2 r}(\chi)=0(r \geq 0)
$$




$$
C_{2 r-1}(\chi)=-\sum_{m=1}^{r-1} \frac{k^{2 r-2 m}}{(2 r-2 m+1) !} C_{2 m-1}(\chi)-\frac{1}{k(2 r) !} \sum_{m=1}^{k-1} m^{2 r} \chi(m)(r \geq 2) .
$$

If $\chi(-1)=-1$,

$$
\begin{gathered}
C_{0}(\chi)=\frac{1}{k} \sum_{m=1}^{k-1} m \chi(m), \quad C_{2 r-1}(\chi)=0(r \geq 1), \\
C_{2 r}(\chi)=-\sum_{m=0}^{r-1} \frac{k^{2 r-2 m}}{(2 r-2 m+1) !} C_{2 m}(\chi)+\frac{1}{k(2 r+1) !} \sum_{m=1}^{k-1} m^{2 r+1} \chi(m)(r \geq 1) .
\end{gathered}
$$

Proof. The first formula of (2.4) and the last formula of (2.5) show that for $r \geq 1$ :

$$
C_{2 r}(\chi)=p_{2 r}(0, \chi)=0 \text { if } \chi(-1)=+1 \text {, and } C_{2 r-1}(\chi)=p_{2 r-1}(0, \chi)=0 \text { if } \chi(-1)=-1 \text {. }
$$

According to the last formula of (2.1), we have

$$
C_{0}(\chi)=\frac{1}{k} \sum_{m=1}^{k-1} m \chi(m) \text {, and } C_{1}(\chi)=\frac{k}{2} C_{0}(\chi)-\frac{1}{2 k} \sum_{m=1}^{k-1} m^{2} \chi(m) .
$$

Also for $\alpha \geq 2$,

$$
C_{\alpha}(\chi)=\frac{1}{k} \int_{0}^{k} x p_{\alpha-1}(x, \chi) d x .
$$

Integration by parts $\alpha-1$ times gives us

$$
C_{\alpha}(\chi)=\left(\sum_{m=2}^{\alpha} \frac{(-1)^{m} k^{m}}{m !} C_{\alpha-m+1}(\chi)+\frac{(-1)^{\alpha+1}}{\alpha !} \int_{0}^{k} x^{\alpha} p_{0}(x, \chi) d x\right) .
$$

But

$$
\int_{0}^{k} x^{\alpha} p_{0}(x, \chi) d x=C_{\alpha}(\chi) \frac{k^{\alpha+1}}{\alpha+1}-\frac{1}{\alpha+1} \sum_{m=1}^{k-1} m^{\alpha+1} \chi(m),
$$

from which we find

$$
C_{\alpha}(\chi)=\sum_{m=2}^{\alpha+1} \frac{(-1)^{m} k^{m-1}}{m !} C_{\alpha-m+1}(\chi)+\frac{(-1)^{\alpha}}{k(\alpha+1) !} \sum_{m=1}^{k-1} m^{\alpha+1} \chi(m) .
$$

If $\chi(-1)=+1$, to obtain the formulas (3.1), we simply take $\alpha=2 r-1$ with $(r \geq 2)$ in (3.3), taking into consideration that $C_{2 r}(\chi)=0$.

If $\chi(-1)=-1$, to obtain the formulas (3.2), we simply take $\alpha=2 r$ with $(r \geq 1)$ in (3.3), taking into consideration that $C_{2 r-1}(\chi)=0$. The theorem is proved. 
Corollary 3.2 For any primitive character $\chi$ modulo $k \geq 3$, we have $C_{r}(\bar{\chi})=\overline{C_{r}(\chi)}$ for $(r \geq 0)$.

Proof. The result follows directly by using induction on $r$.

Example 3.3 1. Let $\chi_{3}$ and $\chi_{4}$ be the non-principal Dirichlet characters modulo 3 and 4 , respectively. Then we have $C_{2 r+1}\left(\chi_{3}\right)=C_{2 r+1}\left(\chi_{4}\right)=0(r \geq 0)$, since $\chi_{3}$ and $\chi_{4}$ are odd characters. Also, it follows by using Theorem 3.1 the following table:

\begin{tabular}{|c|c|c|c|c|c|c|c|}
\hline & $C_{0}$ & $C_{2}$ & $C_{4}$ & $C_{6}$ & $C_{8}$ & $C_{10}$ & $C_{12}$ \\
\hline$\chi_{3}$ & $-\frac{1}{3}$ & $\frac{1}{9}$ & $-\frac{1}{36}$ & $\frac{7}{1080}$ & $-\frac{809}{544320}$ & $\frac{1847}{5443200}$ & $-\frac{7943}{102643200}$ \\
\hline$\chi_{4}$ & $-\frac{1}{2}$ & $\frac{1}{4}$ & $-\frac{5}{48}$ & $\frac{61}{1440}$ & $-\frac{277}{16128}$ & $\frac{50521}{7257600}$ & $-\frac{540553}{19160040}$ \\
\hline
\end{tabular}

Table 1. The first values of $C_{r}(r=0,2, \ldots, 12)$ for $\chi_{3}$ and $\chi_{4}$.

2. Let $\chi_{1,5}, \chi_{2,5}$, and $\chi_{3,5}$ be the Dirichlet character modulo 5 such that $\chi_{1,5}(2)=-1$, $\chi_{2,5}(2)=i$, and $\chi_{3,5}=\overline{\chi_{2,5}}$. Then we have $C_{2 r}\left(\chi_{1,5}\right)=0(r \geq 0)$, since $\chi_{1,5}$ is even character. Also, by using Theorem 3.1 we obtain the following:

\begin{tabular}{|c|c|c|c|c|c|c|c|}
\hline & $C_{1}$ & $C_{3}$ & $C_{5}$ & $C_{7}$ & $C_{9}$ & $C_{11}$ & $C_{13}$ \\
\hline$\chi_{1,5}$ & $-\frac{2}{5}$ & $\frac{1}{3}$ & $-\frac{67}{300}$ & $\frac{361}{2520}$ & $-\frac{412751}{4536000}$ & $\frac{1150921}{19958400}$ & $-\frac{568591843}{15567552000}$ \\
\hline
\end{tabular}

Table 2. The first values of $C_{r}(r=1,3, \ldots, 13)$ for $\chi_{1,5}$.

On the other hand, since $\chi_{2,5}$ and $\chi_{3,5}$ are odd, we have $C_{2 r+1}\left(\chi_{2,5}\right)=C_{2 r+1}\left(\chi_{3,5}\right)=0(r \geq$ $0)$. Finally, Theorem 3.1 and Corollary 3.2 allow us to get

\begin{tabular}{|c|c|c|c|c|c|c|}
\hline & $C_{0}$ & $C_{2}$ & $C_{4}$ & $C_{6}$ & $C_{8}$ & $C_{10}$ \\
\hline$\chi_{2,5}$ & $-\frac{3}{5} \mp \frac{1}{5} i$ & $\frac{2}{5} \pm \frac{1}{5} i$ & $-\frac{37}{150} \mp \frac{43}{300} i$ & $\frac{139}{900} \pm \frac{169}{1800} i$ & $-\frac{4913}{50400} \mp \frac{6047}{100800} i$ & $\frac{279763}{4536000} \pm \frac{345433}{9072000} i$ \\
\hline$\chi_{3,5}$ & &
\end{tabular}

Table 3. The first values of $C_{r}(r=0,2, \ldots, 10)$ for $\chi_{2,5}$ and $\chi_{3,5}$.

Theorem 3.4 Let $\chi$ be a primitive character modulo $k \geq 3$. Then for all $x \in \mathbb{R}_{+}$we have

1. If $\chi(-1)=+1$ and $r \geq 1$ :

$$
\left|p_{2 r-1}(x, \chi)\right| \leq\left|C_{2 r-1}(\chi)\right|
$$

2. If $\chi(-1)=-1$ and $r \geq 0$ :

$$
\left|p_{2 r}(x, \chi)\right| \leq\left|C_{2 r}(\chi)\right|
$$


Proof. 1. Let $\chi(-1)=+1, r \geq 1$ and $x \in \mathbb{R}_{+}$. Then from the first formula of (2.4) we have

$$
\left|p_{2 r-1}(x, \chi)\right|=\frac{|E(\chi)|}{\pi}\left(\frac{k}{2 \pi}\right)^{2 r-1}\left|\sum_{n=1}^{+\infty} \frac{\bar{\chi}(n)}{n^{2 r}} \cos \left(\frac{2 n \pi x}{k}\right)\right| .
$$

If we put $\bar{\chi}(n)=a(n)+i b(n)$ for $(n \geq 1)$, then we get

$$
\sum_{n=1}^{+\infty} \frac{\bar{\chi}(n)}{n^{2 r}} \cos \left(\frac{2 n \pi x}{k}\right)=f(x)+i g(x)
$$

where

$$
f(x)=\sum_{n=1}^{+\infty} \frac{a(n)}{n^{2 r}} \cos \left(\frac{2 n \pi x}{k}\right), \text { and } g(x)=\sum_{n=1}^{+\infty} \frac{b(n)}{n^{2 r}} \cos \left(\frac{2 n \pi x}{k}\right),
$$

from which we can write

$$
\left|p_{2 r-1}(x, \chi)\right|=\frac{|E(\chi)|}{\pi}\left(\frac{k}{2 \pi}\right)^{2 r-1} \sqrt{(f(x))^{2}+(g(x))^{2}} .
$$

On the other hand we have

$$
\begin{aligned}
\left|p_{2 r-1}(0, \chi)\right| & =\frac{|E(\chi)|}{\pi}\left(\frac{k}{2 \pi}\right)^{2 r-1}\left|\sum_{n=1}^{+\infty} \frac{\bar{\chi}(n)}{n^{2 r}}\right|=\frac{|E(\chi)|}{\pi}\left(\frac{k}{2 \pi}\right)^{2 r-1}\left|\sum_{n=1}^{+\infty} \frac{a(n)}{n^{2 r}}+i \sum_{n=1}^{+\infty} \frac{b(n)}{n^{2 r}}\right| \\
& =\frac{|E(\chi)|}{\pi}\left(\frac{k}{2 \pi}\right)^{2 r-1} \sqrt{\left(\sum_{n=1}^{+\infty} \frac{a(n)}{n^{2 r}}\right)^{2}+\left(\sum_{n=1}^{+\infty} \frac{b(n)}{n^{2 r}}\right)^{2} .}
\end{aligned}
$$

Now, we wish to prove that

$$
(f(x))^{2} \leq\left(\sum_{n=1}^{+\infty} \frac{a(n)}{n^{2 r}}\right)^{2}, \text { and }(g(x))^{2} \leq\left(\sum_{n=1}^{+\infty} \frac{b(n)}{n^{2 r}}\right)^{2} .
$$

Let $X=\sum_{n=1}^{+\infty} \frac{a(n)}{n^{2 r}}$. Then $(f(x))^{2}-X^{2}=(f(x)-X)(f(x)+X)$, so that

$$
f(x)-X=\sum_{n=1}^{+\infty}\left(\frac{a(n)}{n^{2 r}}\left(\cos \left(\frac{2 n \pi x}{k}\right)-1\right)\right), f(x)+X=\sum_{n=1}^{+\infty}\left(\frac{a(n)}{n^{2 r}}\left(\cos \left(\frac{2 n \pi x}{k}\right)+1\right)\right)
$$

which are absolutely convergent series, i.e.

$$
(f(x))^{2}-X^{2}=\sum_{n=1}^{+\infty}\left\{\left(\frac{a(n)}{n^{2 r}}\right)^{2}\left(\cos ^{2}\left(\frac{2 n \pi x}{k}\right)-1\right)\right\} \leq 0
$$

By the same, we can show that $(g(x))^{2} \leq\left(\sum_{n=1}^{+\infty} \frac{b(n)}{n^{2 r}}\right)^{2}$. Thus

$$
\left|p_{2 r-1}(x, \chi)\right| \leq\left|p_{2 r-1}(0, \chi)\right|=\left|C_{2 r-1}(\chi)\right| \text {. }
$$


2. Let $\chi(-1)=-1$ and $r \geq 0$. Then by the same reasoning as above we get the second formula.

Now, let us rewrite the formula (1.2) by using the numbers $C_{r}(\chi)$.

Theorem 3.5 Let $\chi$ be a primitive character modulo $k \geq 3$.

1. If $\chi(-1)=+1$ and $r \geq 1$ then:

$$
L(2 r, \bar{\chi})=(-1)^{r} \frac{\pi}{E(\chi)}\left(\frac{2 \pi}{k}\right)^{2 r-1} C_{2 r-1}(\chi) .
$$

2. If $\chi(-1)=-1$ and $r \geq 0$ then:

$$
L(2 r+1, \bar{\chi})=(-1)^{r+1} \frac{\pi}{E(\chi)}\left(\frac{2 \pi}{k}\right)^{2 r} C_{2 r}(\chi) .
$$

Proof. This follows directly by taking $x=0$ in the first formula of (2.4) and $x=0$ in the last formula of (2.5), taking into consideration that $p_{r}(0, \chi)=C_{r}(\chi)$.

The following corollary gives the relationship between the numbers $C_{r-1}(\chi)$ and the generalized Bernoulli numbers $B_{r}(\chi)$.

Corollary 3.6 Let $\chi$ be a primitive character modulo $k \geq 3$.

1. If $\chi(-1)=+1$ and $r \geq 1$ then:

$$
C_{2 r-1}(\chi)=\frac{-1}{(2 r) !} B_{2 r}(\chi)
$$

2. If $\chi(-1)=-1$ and $r \geq 0$ then:

$$
C_{2 r}(\chi)=\frac{1}{(2 r+1) !} B_{2 r+1}(\chi) .
$$

Proof. This follows directly from Theorem 3.5 and the formula (1.2).

The above corollary allows us to rewrite the formula (1.1) as:

Corollary 3.7 Let $\chi$ be a primitive character modulo $k \geq 3$.

1. If $\chi(-1)=+1$ and $r \geq 0$ then:

$$
L(-(2 r+1), \chi)=(2 r+1) ! C_{2 r+1}(\chi) .
$$

2. If $\chi(-1)=-1$ and $r \geq 0$ then:

$$
L(-2 r, \chi)=-(2 r) ! C_{2 r}(\chi) .
$$

As an application, the following theorem gives explicit formulas for sums related to the generalized Bernoulli numbers.

Theorem 3.8 Let $\chi$ be a primitive character modulo $k \geq 3$.

1. If $\chi(-1)=+1$ and $r \geq 1$ then: 


$$
\sum_{m=1}^{r} \frac{k^{2 r-2 m}}{2 r-2 m+1}\left(\begin{array}{c}
2 r \\
2 m
\end{array}\right) B_{2 m}(\chi)=\frac{1}{k} \sum_{m=1}^{k} m^{2 r} \chi(m) .
$$

2. If $\chi(-1)=-1$ and $r \geq 0$ then:

$$
\sum_{m=0}^{r} \frac{k^{2 r-2 m}}{2 r-2 m+1}\left(\begin{array}{c}
2 r+1 \\
2 m+1
\end{array}\right) B_{2 m+1}(\chi)=\frac{1}{k} \sum_{m=1}^{k} m^{2 r+1} \chi(m),
$$

Proof. 1. Let $\chi(-1)=+1$ and $r \geq 1$. Then from the formulas (3.1) we have

$$
C_{2 r-1}(\chi)=-\sum_{m=1}^{r-1} \frac{k^{2 r-2 m}}{(2 r-2 m+1) !} C_{2 m-1}(\chi)-\frac{1}{k(2 r) !} \sum_{m=1}^{k-1} m^{2 r} \chi(m) .
$$

The Corollary 3.6 allows us to write

$$
B_{2 r}(\chi)=-\sum_{m=1}^{r-1} \frac{k^{2 r-2 m}(2 r) !}{(2 m) !(2 r-2 m+1) !} B_{2 m}(\chi)+\frac{1}{k} \sum_{m=1}^{k-1} m^{2 r} \chi(m),
$$

so

$$
B_{2 r}(\chi)+\sum_{m=1}^{r-1} \frac{k^{2 r-2 m}}{(2 r-2 m+1)} \frac{(2 r) !}{(2 m) !(2 r-2 m) !} B_{2 m}(\chi)=\frac{1}{k} \sum_{m=1}^{k-1} m^{2 r} \chi(m),
$$

from which

$$
\sum_{m=1}^{r} \frac{k^{2 r-2 m}}{2 r-2 m+1}\left(\begin{array}{c}
2 r \\
2 m
\end{array}\right) B_{2 m}(\chi)=\frac{1}{k} \sum_{m=1}^{k} m^{2 r} \chi(m) .
$$

2. Let $\chi(-1)=-1$ and $r \geq 0$. Then similarly we get the second formula. This proves the theorem.

As another application, the following theorem gives asymptotic formulas for $L(s, \chi)$ in terms of the generalized Bernoulli numbers.

Theorem 3.9 Let $\chi$ be a primitive character modulo $k \geq 3$ and let $L(s, \chi)$ be the Dirichlet $L$ function corresponding to $\chi$. Let $N$ and $r$ be positive integers such that $R(s)=\sigma>1-2 r$ if $\chi(-1)=+1$ and $\sigma>-2 r$ if $\chi(-1)=-1$. Then

$$
\begin{gathered}
L(s, \chi)=\sum_{n=1}^{k N} \frac{\chi(n)}{n^{s}}+\sum_{m=1}^{r} \frac{B_{2 m}(\chi)}{(2 m) !} \prod_{j=0}^{2 m-2}\left(\frac{s+j}{(k N)^{s+1}}\right)+R_{1}(s) \text { if } \chi(-1)=+1, \\
L(s, \chi)=\sum_{n=1}^{k N} \frac{\chi(n)}{n^{s}}-s \frac{B_{1}(\chi)}{(k N)^{s}}-\sum_{m=1}^{r} \frac{B_{2 m+1}(\chi)}{(2 m+1) !} \prod_{j=0}^{2 m-1}\left(\frac{s+j}{(k N)^{s+1}}\right) \\
+R_{2}(s) \text { if } \chi(-1)=-1,
\end{gathered}
$$


where

$$
\left|R_{1}(s)\right| \leq\left|T_{2 r-1}(\chi)\right| \times \frac{|s+2 r-1|}{\sigma+2 r-1}, \text { and }\left|R_{2}(s)\right| \leq\left|T_{2 r}(\chi)\right| \times \frac{|s+2 r|}{\sigma+2 r},
$$

with

$$
T_{2 r-1}(\chi)=C_{2 r-1}(\chi) \frac{s(s+1) \cdots(s+2 r-2)}{(k N)^{s+2 r-1}}, T_{2 r}(\chi)=C_{2 r}(\chi) \frac{s(s+1) \cdots(s+2 r-1)}{(k N)^{s+2 r}} .
$$

Proof. First of all we can write

$$
L(s, \chi)=\sum_{n=1}^{k N} \frac{\chi(n)}{n^{s}}+\sum_{n>k N}^{+\infty} \frac{\chi(n)}{n^{s}} .
$$

It follows by using [5, Theorem 1.3] that:

$$
\sum_{n>k N}^{+\infty} \frac{\chi(n)}{n^{s}}=s \int_{k N}^{+\infty} \frac{S(t, \chi)}{t^{s+1}} d t=-s \frac{C_{0}(\chi)}{(k N)^{s}}+s \int_{k N}^{+\infty} \frac{p_{0}(t, \chi)}{t^{s+1}} d t
$$

If $\chi(-1)=+1$, then $C_{2 r}(\chi)=0(r \geq 0)$. Integrating by parts $2 r-1$ times the integral in the right hand side of (3.6), taking into consideration that $p_{r}(k N, \chi)=C_{r}(\chi)$, we obtain:

$$
\begin{aligned}
L(s, \chi)=\sum_{n=1}^{k N} \frac{\chi(n)}{n^{s}}-C_{1}(\chi) \frac{s}{(k N)^{s+1}}-C_{3}(\chi) \frac{s(s+1)(s+2)}{(k N)^{s+3}}-\cdots \\
-C_{2 r-1}(\chi) \frac{s(s+1)(s+2) \cdots(s+2 r-2)}{(k N)^{s+2 r-1}} \\
+s(s+1)(s+2) \cdots(s+2 r-1) \int_{k N}^{+\infty} \frac{p_{2 r-1}(t, \chi)}{t^{s+2 r}} d t .
\end{aligned}
$$

Now Corollary 3.6 and the formula (3.7) imply the formula (3.4) with:

According to Theorem 3.4, we have

$$
R_{1}(s)=s(s+1)(s+2) \cdots(s+2 r-1) \int_{k N}^{+\infty} \frac{p_{2 r-1}(t, \chi)}{t^{s+2 r}} d t .
$$

$$
\begin{aligned}
R_{1}(s) & \leq|s(s+1)(s+2) \cdots(s+2 r-1)| \times\left|C_{2 r-1}(\chi)\right|\left|\int_{k N}^{+\infty} \frac{1}{t^{s+2 r}} d t\right| \\
& =\left|\frac{s(s+1)(s+2) \cdots(s+2 r-1) C_{2 r-1}(\chi)}{(k N)^{s+2 r-1}}\right| \frac{|s+2 r-1|}{\sigma+2 r-1} \\
& =\left|T_{2 r-1}(\chi)\right| \times \frac{|s+2 r-1|}{\sigma+2 r-1} .
\end{aligned}
$$

If $\chi(-1)=-1$, then $C_{2 r-1}(\chi)=0(r \geq 1)$. Integrating by parts $2 r$ times the integral in the right hand side of (3.6), taking into consideration that $p_{r}(k N, \chi)=C_{r}(\chi)$, we obtain: 


$$
\begin{aligned}
L(s, \chi)=\sum_{n=1}^{k N} \frac{\chi(n)}{n^{s}}-C_{0}(\chi) \frac{s}{(k N)^{s+1}}-C_{2}(\chi) \frac{s(s+1)}{(k N)^{s+2}}-\cdots \\
-C_{2 r}(\chi) \frac{s(s+1)(s+2) \cdots(s+2 r)}{(k N)^{s+2 r}} \\
+s(s+1)(s+2) \cdots(s+2 r) \int_{k N}^{+\infty} \frac{p_{2 r}(t, \chi)}{t^{s+2 r+1}} d t
\end{aligned}
$$

Now Corollary 3.6 and the formula (3.8) imply the formula (3.5) with:

$$
R_{2}(s)=s(s+1)(s+2) \cdots(s+2 r) \int_{k N}^{+\infty} \frac{p_{2 r}(t, \chi)}{t^{s+2 r+1}} d t
$$

According to Theorem 3.4, we have

This completes the proof.

$$
\begin{aligned}
R_{2}(s) & \leq|s(s+1)(s+2) \cdots(s+2 r)| \times\left|C_{2 r}(\chi)\right|\left|\int_{k N}^{+\infty} \frac{1}{t^{s+2 r+1}} d t\right| \\
& =\left|\frac{s(s+1)(s+2) \cdots(s+2 r-1) C_{2 r}(\chi)}{(k N)^{s+2 r}}\right| \frac{|s+2 r|}{\sigma+2 r} \\
& =\left|T_{2 r}(\chi)\right| \times \frac{|s+2 r|}{\sigma+2 r} .
\end{aligned}
$$

Remark 3.10 From [3, p.37] we have

$$
L(s, \chi)=\prod_{p \mid k}\left(1-\frac{\chi^{*}(p)}{p^{s}}\right) L\left(s, \chi^{*}\right),
$$

where $\chi^{*}$ is the unique primitive character which induces $\chi$. Thus $L(s, \chi)$ can be expressed in terms of $L\left(s, \chi^{*}\right)$, so one can use this fact to generalize above theorem to an arbitrary character $\chi$.

Remark 3.11 One can use the first formula of (2.4) and the last formula of (2.5) to get another upper bound of $R_{1}(s)$ and of $R_{2}(s)$ as follows:

and

$$
R_{1}(s) \leq \frac{2|s| \sqrt{k} \zeta(2 r)}{(2 r+\sigma-1)(2 \pi)^{2 r}(k N)^{\sigma}} \prod_{j=1}^{2 r-1}\left(\frac{|s+j|}{N}\right),
$$

\section{CONCLUSION}

$$
R_{2}(s) \leq \frac{2|s| \sqrt{k} \zeta(2 r+1)}{(2 r+\sigma)(2 \pi)^{2 r+1}(k N)^{\sigma}} \prod_{j=1}^{2 r}\left(\frac{|\mathrm{s}+\mathrm{j}|}{\mathrm{N}}\right) .
$$

In this paper, we define complex numbers associated with a primitive Dirichlet character $\chi$, and we use them to rewrite some known results as formulas (1.1) and (1.2). Also, we use them to give explicit formulas for sums related to the generalized Bernoulli numbers, as shown by the Theorem 3.8, and to give asymptotic formulas for $L(s, \chi)$ in terms of the generalized Bernoulli numbers, as shown by the Theorem 3.9.

Acknowledgements: The authors would like to thank the anonymous referee for their careful reading and valuable suggestions which certainly improved the readability of this paper. 


\section{REFERENCES}

[1] T. M. Apostol, Introduction to Analytic Number Theory, Springer-Verlage, New York (1976).

[2] T. Arakawa, T. Ibukiyama, and M. Kaneko, Bernoulli Numbers and Zeta Functions, Springer Japan (2014).

[3] H. Davenport, Multiplicative Number Theory, Springer-Verlage, New York (1980).

[4] D. Davies and C.B. Haselgrove, "Evaluation of Dirichlet L-Functions", Proc. Roy. Soc. Ser.

A, 264, 122-132 (1961).

[5] H.L. Montgomery and R.C. Vaghan, Multiplicative Number Theory I. Classical Theory, Cambridge University Press (2006).

Received June 8, 2020 